\section{ABCs of drug resistance}

Multidrug resistance resulting from the activity of members of the ABC (ATP-binding cassette) family of membrane transporters, which actively pump many types of anticancer drugs out of cancer cells, is a significant barrier to effective cancer chemotherapy. However, efforts to

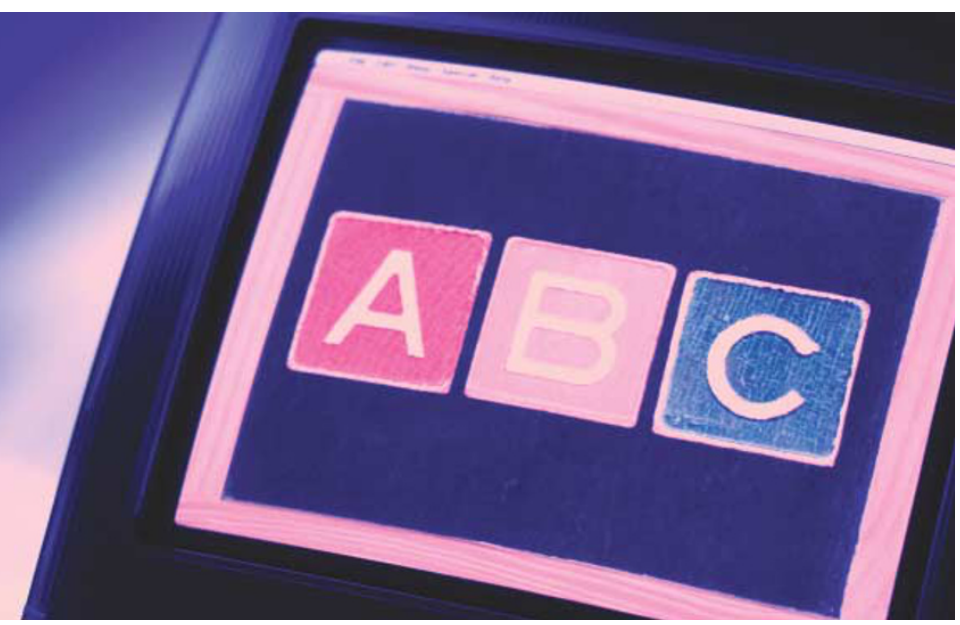

overcome this problem have been hampered by limited knowledge of many members of the ABC transporter family. Now, as reported in Cancer Cell, Szakács et al. have coupled expression data for all human $\mathrm{ABC}$ transporters in cancer cells with information on the sensitivity of the cells to various anticancer drugs, providing a valuable tool for improving understanding of drug resistance mediated by these proteins.

Of the 48 human $\mathrm{ABC}$ transporters, only $\sim 10$ have been associated with cancer-drug resistance, such as the archetypal multidrug transporter ABCB1 (also known as MDR1 and P-glycoprotein). However, the high degree of sequence similarity between these $\mathrm{ABC}$ transporters suggested that further members might also export cancer drugs. So, Szakács and Annereau in the Gottesman lab, in collaboration with the Weinstein group, set out to explore this possibility by characterizing the expression of $\mathrm{ABC}$ transporters in a set of cancer cells whose responses to a large number of compounds are well-characterized: the panel of 60 cancer cell lines at the National
Cancer Institute, which have been used to screen $>100,000$ compounds.

By using real-time PCR assays, which are more specific and sensitive than microarrays, to quantify the levels of messenger RNA of all 48 human ABC transporters, the authors obtained precise correlations between $\mathrm{ABC}$ transporter expression and known patterns of drug activity for 1,429 compounds across the 60 cancer cell lines. As expected, good agreement was found between expression of ABCB1 and reduced cellular sensitivity to anticancer drugs that are known to be substrates for this transporter. Furthermore, using this method, several compounds that were not previously known to be substrates of ABCB1 were identified as potential substrates of this transporter, and these predictions were validated with follow-up experiments. And unexpectedly, some compounds were predicted — and subsequently confirmed — to have their anticancer activity potentiated by $A B C B 1$, rather than antagonized; these compounds might serve as leads for drug development.

In total, the data revealed 131 drug-transporter pairs in which

\section{Preventing the bubble bursting?}

5-lipoxygenase (5-LO) - an enzyme that is crucial for the synthesis of a class of proinflammatory lipids known as leukotrienes has been identified as a potential therapeutic target for preventing life-threatening arterial ruptures that are often linked with atherosclerosis. Reporting in Nature Medicine, Funk and colleagues provide several lines of converging evidence that the 5-LO cascade is involved in the expression of inflammatory proteins in cells associated with atherosclerosis and contributes to the pathogenesis of aortic aneurysms.

An aneurysm is an abnormal enlargement or bulging of an artery that results from a weakening of the media, the smoothmuscle-cell-rich middle layer of the artery wall. The consequences of an aneurysm bursting can be extremely serious, and so surgery is typically used as a prophylactic measure once the aneurysm reaches a certain size; however, surgery is not without risk itself.
Several previous studies raised the possibility that 5-LO might have a role in cardiovascular disease, and so Funk and colleagues investigated the involvement of the 5-LO pathway in the formation of aneurysms induced by an atherogenic diet in mice that were rendered susceptible to atherosclerosis by knocking out the gene that encodes apolipoprotein $\mathrm{E}$ (APOE). Macrophages that expressed 5-LO were abundant in the adventitia (the outermost layer of the arterial wall) of these mice, which showed a high incidence of aneurysms in the aorta. However, mice in which the genes that encode both APOE and 5-LO were knocked out showed a much lower incidence and extent of aortic aneurysm development in the absence of changes in the degree of atherosclerosis. Furthermore, the activity of the metalloprotease MMP2, which is thought to be required for the remodelling involved in aneurysm formation, was significantly reduced in these mice compared with Apoe knockout mice.
A more detailed investigation of the 5-LO cascade using mouse and human cells revealed that the leukotriene $\mathrm{LTD}_{4}$ activates the expression of chemokines, including the macrophage inflammatory proteins MIP1- $\alpha$ in macrophages and MIP2 in endothelial cells, indicating a mechanism by which the 5-LO pathway might promote inflammation and aneurysm formation.

Taken together, these findings indicate that expression of 5-LO in adventitial macrophages generates leukotrienes, which in turn leads to the release of inflammatory cytokines, and, through indirect mechanisms, protease activity. The existence of these 5-LO-dependent inflammatory circuits highlights the possibility of using drugs that directly block aneurysm progression in susceptible patients as a promising alternative to surgical intervention.

\section{Alison Rowan}

(6) References and links ORIGINAL RESEARCH PAPER Zhao, L. et al. The 5-lipoxygenase pathway promotes pathogenesis of hyperlipidemia-dependent aortic aneurysm. Nature Med. 10, 966-973 (2004)

FURTHER READING Palinski, W. Aneurysms: leukotrienes weaken aorta from the outside. Nature Med.10, 896-898 (2004) 
transporter expression correlated with resistance to the drug. As well as transporters known to be associated with drug resistance, several transporters that were not previously implicated in drug resistance were identified; again, follow-up experiments confirmed some of these predictions.

So, overall, the database created by the authors provides a resource for identifying transporters whose expression confers drug resistance, and compounds whose effects are antagonized, unaffected or even potentiated by transporter expression, which will be useful in developing strategies to address the problem of multidrug resistance. The database will also be valuable for future data mining to aid in studies of the function of the many $\mathrm{ABC}$ transporters that are not well characterized.

Peter Kirkpatrick

(2) References and links ORIGINAL RESEARCH PAPER Szakács, G. et al. Predicting drug sensitivity and resistance: profiling ABC transporter genes in cancer cells. Cancer Cell 6, 129-137 (2004)

FURTHER READING Gottesman, M. M. et al.

Multidrug resistance in cancer: role of

ATP-dependent transporters. Nature Rev. Cancer 2, 48-58 (2002)

WEB SITE Database: http://discover.nci.nih.gov/ abc/2004_cancercell_abstract.jsp

\section{APOPTOSIS}

\section{Bid for death}

Death and taxes - two things that are famously certain in life. However, as we learn more, it seems that although death might be more complicated than taxes, it is perhaps not as inevitable! Programmed cell death, known as apoptosis, is a crucial process for tissue homeostasis, but uncontrolled cell death is associated with several human pathologies, such as neurodegenerative diseases and ischaemic injuries. In the August issue of Chemistry \& Biology, Pellecchia and colleagues demonstrate the power of a multidisciplinary NMR-based approach in the rational design of a series of small-molecule antagonists that bind to the pro-apoptotic molecule Bid.

A number of protein families regulate apoptosis, some of which promote death, whereas others maintain life. Although several smallmolecule inhibitors of anti-apoptotic proteins have been reported so far, none have been discovered for pro-apoptotic members, including Bid, a key member of the BCL2 protein family. Mice deficient in Bid show resistance to cell death in models of liver injury and stroke, and the molecule is also implicated in mouse models of amyotrophic lateral sclerosis. As the molecular basis for its function is unknown, Bid represents a challenging target for the development of therapeutic agents.

Even though the crystal structure of Bid is unsolved, NMR spectroscopy has revealed the presence of a deep hydrophobic crevice on the surface of the protein, in a region that is conserved between mouse and human Bid. The authors screened a fragment library that is made up of low-molecular-mass compounds that represent a selection of the substructures often found in drugs, and which are amenable to subsequent chemistry. Because of the simplicity of the structures, a few hundred derivatives are sufficient to represent great diversity; however, due to their limited size, and the consequent limited number of possible interactions with a given protein, many of the fragments will show low affinity for the target.

To overcome this problem, solution NMR was used as a screening method, because it allows the detection of very weak binders. The authors named this method structure-activity relationships by interligand nuclear Overhauser effect (SAR by ILOEs); by combining this with molecular modelling and synthetic chemistry they were able to rationally design a series of 4-phenylsulphanyl-phenylamine derivatives capable of occupying the hydrophobic crevice of Bid.



In cell-based assays, one of the newly synthesized compounds, BI-6C9, was able to bind to Bid and dramatically reduce production of mitochondrial SMAC, a downstream Biddependent event. Certainly, this compound will be useful in deciphering the mechanism of action of Bid within the complex process of apoptosis; with further optimization, BI-6C9 might also provide a starting point for the development of drug candidates for diseases associated with uncontrolled cell death. In addition, the authors have demonstrated the utility of multidisciplinary SAR by ILOE in tackling challenging drug targets, and possible future applications could include developing inhibitors of protein-protein, or protein-nucleic acid, interactions.

Melanie Brazil

(4) References and links ORIGINAL RESEARCH PAPER Becattini, B. et al. Targeting apoptosis via chemical design: inhibition of Bid-induced cell death by small organic molecules. Chem. Biol. 11, 1107-1117 (2004) FURTHER READING

Rees, D. C. et al. Fragment-based lead discovery. Nature Rev. Drug Discov. 3, 660-672 (2004) | Pellecchia, M., Sem, D. S. \& Wuthrich, K. NMR in drug discovery. Nature Rev. Drug Discov. 1, 211-219 (2002) | Reed, J. C. Apoptosis-based therapies. Nature Rev. Drug Discov. 1, 111-121 (2002) 